\title{
BMJ Open Effects of vaccines in protecting against Ebola virus disease: protocol for a systematic review
}

\author{
Lindi Mathebula, ${ }^{\odot}$ Duduzile Edith Ndwandwe, Elizabeth Pienaar, \\ Charles Shey Wiysonge
}

To cite: Mathebula L, Ndwandwe DE, Pienaar E, et al. Effects of vaccines in protecting against Ebola virus disease: protocol for a systematic review. BMJ Open 2019;9:e029617. doi:10.1136/ bmjopen-2019-029617

- Prepublication history for this paper is available online. To view these files, please visit the journal online (http://dx.doi. org/10.1136/bmjopen-2019029617).

Received 1 February 2019 Revised 7 May 2019 Accepted 31 May 2019
D) Check for updates

(C) Author(s) (or their employer(s)) 2019. Re-use permitted under CC BY-NC. No commercial re-use. See rights and permissions. Published by BMJ.

Cochrane South Africa, South African Medical Research Council, Tygerberg, South Africa

Correspondence to Lindi Mathebula; lindi.mathebula@mrc.ac.za

\section{ABSTRACT}

Introduction Ebola virus disease is one of the most devastating infectious diseases in the world with up to $90 \%$ case fatality observed. There are at least 13 candidate vaccines developed and being tested to prevent the occurrence of the Ebola virus disease. While none of these candidate vaccines has received regulatory approval for use, one candidate vaccine (rVSV $\triangle$ G-ZEBOV-GP) has been granted access for emergency use. Two other candidate vaccines (GamEvac-Combi and Ad5-EBOV) have been licensed for emergency use in their countries of origin. The objective of this systematic review is to summarise the effects of the Ebola candidate vaccines in humans.

Methods and analysis We will search for potentially eligible studies, with no language or date restrictions, in the Cochrane Central Register of Controlled Trials, PubMed, Scopus, the WHO International Clinical Trial Registry Platform, and reference lists of relevant publications. The Cochrane Database of Systematic Reviews (CDSR) and the Database of Abstracts of Reviews of Effect (DARE) will be searched for related reviews. Two review authors will independently screen search records, assess study eligibility, perform data extraction, and assess the risk of bias; and reconcile their findings. We will pool data from similar studies using Mantel-Haenszel's fixed-effect model. Ethics and dissemination This study is exempted from ethical consideration since the data collected are publicly available and at no point will confidential information from human participants be used. We will disseminate our results through publications in peer-reviewed journals and relevant conferences.

PROSPERO registration number CRD42018110505.

\section{BACKGROUND}

\section{Description of the condition}

Ebola virus disease was first observed in the early 1976 and since then there have been a number of small and large outbreaks. ${ }^{1}$ Ebola virus belongs to a family of viruses known as Filoviridae, genus Ebolavirus where there are currently five species identified under this genus, namely Bundibugyo ebolavirus, Reston ebolavirus, Sudan ebolavirus, Tai forest ebolavirus and Zaire ebolavirus. ${ }^{2}$ B. ebolavirus, $R$. ebolavirus, S. ebolavirus and Tai Forest ebolavirus

\section{Strengths and limitations of this study}

- This review will conduct an extensive search for inclusion of all types of study designs with no language restrictions thereby reducing selection bias.

- A careful selection of studies, quality assessment and data extraction will be done in duplicate to ensure rigorous conduct of the review.

- This review will assess risk of bias from the different types of study designs using relevant Cochrane risk of bias tools.

- Meta-analyses of included studies may not be appropriate to perform due to expected heterogeneity of the studies.

are known to cause severe and often deadly haemorrhagic fever. ${ }^{2}$ The case fatality rate of Ebola virus disease varies by species and could be as high as $90 \% .^{3}$ Ebola virus is normally found in animals known as the fruit bat and transmission occurs through human contact with the tissue of fruit bats; ingestion of fruit bat faecal matter in contaminated water, food and plants and human-to-human transmission through bodily fluids. ${ }^{4}$ Once a human host is infected with Ebola virus, the infection to disease mechanism starts with the virus implanting itself inside the body where it begins to replicate and spread to target organs where it continues to multiply leading to disease. ${ }^{5}$ Ebola virus inside the body is enveloped in a lipid membrane that allows the Ebola virus to enter and spread within the macrophages, monocytes and dendritic cells. When the virus reaches the liver and spleen, it damages the tissues either by direct cytotoxic effect or stimulating the immune system to overproduce toxic agents. ${ }^{45}$ Ebola virus has an incubation period of up to 21 days. Symptoms range from transient flu-like episodes to septic shock and death. ${ }^{6}$

\section{Description of the intervention}

In June 2018, it was reported that there were about 36 completed Ebola vaccine trials 
and 14 ongoing trials at various stages of recruitment. ${ }^{7}$ Currently there are no Ebola vaccines that have received regulatory approval for use; however, the attenuated recombinant vesicular stomatitis virus vector vaccine (rVSVAG-ZEBOV-GP) which has recently completed phase clinical trials has been granted access to the Priority Medicine Scheme by the European Medicine Agency and Breakthrough Therapy designation by the US Food and Drug Administration. ${ }^{8}$ While two other candidate vaccines, recombinant vesicular stomatitis virus-based (rVSV) plus human adenovirus type 5 vaccine (Ad5) prime boost (GamEvac-Combi) and recombinant adenovirus type 5 vector-based Ebola vaccine (Ad5-EBOV), have been licensed for emergency use in Russia and China, respectively. ${ }^{8}$ In addition, there are various candidate vaccines undergoing phases $1-3$ clinical testing. ${ }^{9}$

\section{How the intervention might work}

Many of the Ebola virus candidate vaccines use recombinant vectors based on human adenovirus serotype and chimpanzee adenovirus serotype expressing envelope glycoprotein (GP) of Zaire Ebola virus. ${ }^{81011}$ Ad5-EBOV and Ad5 candidate vaccines are both non-replicative, recombinant human adenovirus serotype 5 expressing envelope GP of Zaire Ebola virus, however Ad5 also expresses envelope GP of Sudan Ebola virus as well. ${ }^{8} 1011$ Adenovirus type-26 vector-based vaccine (Ad26.ZEBOV) is an adenovirus type-26 vector-based vaccine expressing envelope GP of Zaire Ebola virus species which is combined with modified vaccinia virus Ankara-Bavarian nordic (MVABN) Filo-vector vaccine (prime/booster), where MVA-BNFilo expresses four filoviruses nucleoproteins GP of Zaire Ebola virus, Sudan Ebola virus, Tai forest Ebola virus and the Marburg viruses, while recombinant chimpanzee adenovirus type 3-vectored vaccine (ChAd3-EBOZ) is a recombinant chimpanzee adenovirus type 3-vectored vaccine expressing envelope GP of Zaire (Mayinga strain) Ebola virus species also combined MVA-BN filo-vector vaccine (prime/booster). ${ }^{81011}$ Recombinant chimpanzee adenovirus type 3 vaccine (ChAd3) is a non-replicative recombinant chimpanzee adenovirus serotype 3 envelope GP of both Sudan Ebola virus and Zaire Ebola virus. ${ }^{8} 1112$ DNA plasmid vaccines have several candidate vaccines that are either encoded for both the Zaire Ebola virus and Sudan Ebola virus or Marburg viruses. ${ }^{10} 12$ rVSV $\triangle$ G-ZEBOV-GP and Recombinant Vesicular Stomatitis Virus-based Vaccine (VSV N4CT1 EBOVGP1) are both replicative and recombinant vascular stomatitis virus vaccines enveloping GP of Zaire Ebola virus, while GamEvac-Combi (rVSV \& Ad5, prime/boost) is a replicative recombinant vascular stomatitis virus and human adenovirus serotype 5 vaccine expressing envelope for Zaire Ebola virus. ${ }^{811}$ Nanoparticle recombinant Ebola GP vaccine is a nanoparticle recombinant vaccine with or without a matrix-adjuvant that is against the Zaire Ebola virus species, similar to this candidate vaccine is the DNA vaccine (INO-4212) which is delivered using an electroporation is also against past and present Zaire Ebola virus strains. ${ }^{811}$ Lastly, the HPIV3-EbovZ candidate vaccine is a live attenuated human parainfluenza virus type 3 vectored expressing GP of Zaire Ebola virus. Immune responses from the use of these vaccine is observed in the follow-up period of which the longest interval has been at least 12 months. ${ }^{8}$

\section{Why it is important to do this review}

There have been several trials investigating the effects of candidate Ebola vaccines including a systematic review of Ebola vaccine development which reviewed Ebola vaccine studies to assess factors associated with antibody response variability in humans, ${ }^{13}$ However there is no collective evidence of what the effects of the Ebola vaccines are. This review will provide a global view on research conducted on Ebola virus vaccines. In addition, the review will investigate immune responses of participants post vaccination and reasons for (in)efficacy of the vaccines as well as different strategies employed in vaccinating participants.

\section{OBJECTIVES}

To assess and summarise the effects of Ebola candidate vaccines including protection against the Ebola virus disease, the immune response and side effects in humans.

\section{METHODS}

\section{Inclusion criteria}

We provide below the eligibility criteria for selecting studies, including the type of studies, participants, interventions and outcomes.

\section{Types of studies}

Randomised trials, non-randomised trials, case-controlled studies and cohort studies.

\section{Types of participants}

The study will include only human participants regardless of any identifying characteristics such as age, gender, demographics and socio-economic status.

\section{Types of interventions}

Vaccination with any candidate vaccine, including but not limited to the following:

- Attenuated recombinant vesicular stomatitis virus (rVSV) vector vaccine.

- Recombinant adenovirus type 5 vector-based Ebola vaccine (monovalent).

- Recombinant vesicular stomatitis virus-based and human adenovirus type 5 vaccine.

- Recombinant adenovirus type 5 vector-based Ebola vaccine (bivalent).

- Adenovirus type-26 vector-based vaccine (Ad26. ZEBOV) with modified vaccinia virus Ankara-Bavarian nordic (MVA-BN) filo-vector vaccine.

- Recombinant chimpanzee adenovirus type 3 vaccine (monovalent). 
- Recombinant chimpanzee adenovirus type 3 vaccine (bivalent).

- Recombinant chimpanzee adenovirus type 3-vectored vaccine (cAd3-EBO and cAd3-EBOZ) with modified vaccinia virus Ankara-Bavarian nordic (MVA-BN) filovector vaccine.

- Recombinant vesicular stomatitis virus-based vaccine.

- Glycoprotein nanoparticle vaccine (EBOV GP) with or without Matrix-M adjuvant.

- DNA vaccine (INO-4212).

- Live-attenuated human parainfluenza virus type vaccine.

The comparison interventions will be placebo, no intervention or another vaccine.

\section{Types of outcomes}

Primary outcomes

- Incidence of Ebola virus disease.

\section{Secondary outcomes}

- Immunogenicity (measured by virus neutralisation assay).

- Adverse events following vaccination (serious adverse events, minor adverse events).

Adverse events are effects of any severity where there is a possible causal relationship between intervention and the event is identified as reasonable. ${ }^{14}$ Adverse events can be solicited (anticipated) or unsolicited (unexpected). Serious adverse events are adverse effects severe enough to lead to harm, life threatening, requiring hospitalisation or prolonged hospital stay, disability or incapacity, or death of participant attributable to the intervention. ${ }^{14}$

\section{SEARCH METHODS FOR IDENTIFICATION OF STUDIES}

We will search all potential studies (published, unpublished, in press and in progress) with no language or date restrictions on the search strategy.

\section{Electronic searches}

The following databases and sources will be searched to identify published primary studies: Cochrane Central Register of Controlled Trials (CENTRAL); PubMed; and Scopus. We will also search the WHO International Clinical Trial Registry Platform (WHO-ICTRP) for ongoing and completed trials using the search terms "Vaccines" OR "Vaccine" AND "Ebola". Should a trial from the registry be reported complete, the trial record will be checked for reported publications in the "URL to publication" field within the record or using the trial registration ID number to search electronic databases, and by contacting the investigators to ask if results are available for sharing. We provide the proposed search strategy for PubMed in table 1.

\section{Searching other resources}

We will search the CDSR and DARE for related reviews, as well as the reference lists of relevant reviews and other studies.

\begin{tabular}{|c|c|}
\hline Search & Query \\
\hline$\# 1$ & $\begin{array}{l}\text { Search "hemorrhagic fever, ebola"[MeSH] OR } \\
\text { ("hemorrhagic"[All Fields] AND "fever"[All Fields] } \\
\text { AND "ebola) }\end{array}$ \\
\hline \#2 & $\begin{array}{l}\text { Search "ebola hemorrhagic fever"[All Fields] OR } \\
\text { "ebola"[All Fields] OR "ebolavirus"[MeSH Terms] } \\
\text { OR "ebolavirus"[All Fields] }\end{array}$ \\
\hline$\# 3$ & $\begin{array}{l}\text { Search "vaccines"[MeSH Terms] OR "vaccines"[All } \\
\text { Fields] OR "vaccine"[All Fields] OR Vaccination OR } \\
\text { Vaccinated }\end{array}$ \\
\hline$\# 4$ & Search \#1 OR \#2 \\
\hline \#5 & Search \#4 AND \#3 \\
\hline
\end{tabular}

\section{DATA COLLECTION AND ANALYSIS \\ Selection of studies}

Two review authors will independently screen titles and abstracts of all identified studies in the combined search output for potentially eligible studies and exclude clearly irrelevant studies. Full texts will be retrieved for potentially eligible studies and two review authors will independently assess the full text for inclusion into the review using predefined inclusion criteria. The review authors will resolve any disagreements through a discussion, should they fail to resolve the differences, a third review author will pass on judicially for consensus. The study selection process will be recorded in the Preferred Reporting Items for Systematic Reviews and Meta-Analyses (PRISMA) diagram and all potentially eligible studies excluded through full text screening and will be listed in a table describing characteristics of excluded studies with reasons for exclusion.

\section{Data extraction and management}

A data extraction form will be designed and piloted by two review authors for validation. The two review authors will extract data independently and in duplicate from each included study. Two reviewers will meet to compare and combine data extracted. Where there are disagreements, a discussion will be held in order to reach consensus, should an agreement not be reached, a third review author will pass on judicially. The following characteristics of the study will be extracted:

- Study setting, design, sample size, power calculation and trial registration.

- Baseline characteristics including weight, age, sex, HIV status and other comorbidity.

- Intervention vaccine and control vaccines dosages, routes of administration and times of vaccinations.

- Outcomes measures.

- Findings (incidence, immunogenicity, adverse events data).

\section{Assessment of risk of bias in included studies}

Two review authors will independently use the Cochrane Effective Practice and Organisation of Care (EPOC) tool 
to assess risk of bias of included studies in duplicate. Disagreements will be resolved through discussion until consensus is reached. Where necessary a third review author will be consulted to resolve any disagreements. The two review authors will pilot the risk of bias tool on four included studies to ensure consistency in assessing the studies. The EPOC tool has nine listed domains to assess risk of bias for both randomised and non-randomised trials and these include the following: random sequence generation, allocation concealment, blinding of outcome assessors, incomplete outcome data, knowledge of the allocated interventions adequately prevented during the study, selective reporting, baseline characteristics similar, free of contamination, baseline outcomes similar and other biases, all the domains are fully described in EPOC 2017. ${ }^{15}$ The review authors will consider a study as having a low risk of bias if all criteria defined by EPOC are scored as 'yes', an unclear risk of bias will be a study in which one or more criteria are scored as 'unclear', Lastly, a high risk of bias will be a study in which one or more criteria are scored as 'no'. For non-randomised studies of interventions (Cohort and case controlled studies) we will use the ROBINS-I tool to assess the risk of bias which includes three additional domains to those of the EPOC tool namely, confounding, selection of participants into the study and classification of the intervention ${ }^{16}$.

In addition, we will use a funnel plot to assess publication bias.

\section{Measures of treatment effect}

We will analyse all data from included studies using RevMan V.5.3. ${ }^{17}$ We will combine dichotomous data using risk ratio (RR) or ORs with their $95 \%$ Cls and will express continuous outcomes as standardised mean differences with their 95\% Cls. We will attempt to group studies reporting geometric means and ab titres to analyse separately

\section{Unit of analysis issues}

For included studies that have multiple interventions we will use the Cochrane-handbook section 16.5.4 as a guideline to avoid unit-of analysis error and section 16.6.3 for multiple-treatment meta-analysis. ${ }^{18}$ We will analyse the data by combining treatment arms or by splitting the control group so that participants are only included once in the meta-analysis.

\section{Dealing with missing data}

If there is missing data in the included studies, we will assess whether the missing data is related to outcomes and contact the trial authors for more information. We will perform intention to treat analysis to account for missing data of important outcomes.

\section{Assessment of heterogeneity}

We will assess the data extracted to find key differences in the setting, population groups, intervention type, dosages, route of administration, and timing of vaccination. We will also consider degree of risk of bias in the studies, outcome measurement process, and assess variation in treatment effects. ${ }^{18}$ We will inspect the forest plot for overlapping Cls and the following data to determine the level of heterogeneity: $\chi^{2} \mathrm{p}$-value significance of $\leq 0.1$ and an $\mathrm{I}^{2}$ statistical value of more than $50 \%$ as likely heterogeneity.

\section{Data synthesis}

We will stratify analyses by study design and pool data from studies with identical designs, candidate vaccines and outcomes using the fixed-effect Mantel-Haenszel model for meta-analysis. For the meta-analysis of adverse event outcomes, we will pick a maximum of 3 of the most frequent adverse events that were considered to be serious. Should a meta-analysis not be applicable due to heterogeneity of the studies, we will give a narrative report instead. We will also attempt to perform statistical adjustments for sample size and variance for any cluster randomised trials before meta-analysis if appropriate.

\section{Subgroup analysis and investigation of heterogeneity}

We will explore heterogeneity in the studies and perform subgroup analysis by:

- Age of participants that is, children versus adults.

- Pregnancy status that is, pregnant versus not pregnant.

- Comorbidities such as HIV status status that is, pregnant versus not pregnant.

Random-effect analysis will be considered in the meta-analyses if significant statistical heterogeneity in subgroup analyses cannot be explained. We will define significant statistical heterogeneity at the $\chi^{2} p$ value of $\leq 0.1$ and an $\mathrm{I}^{2}$ value of more than $50 \%$.

\section{Sensitivity analysis}

If studies have incomplete outcome data that renders the study to have high risk of bias, we may use imputation and perform sensitivity analysis to assess the impact of the missing data. We will also consider risk of bias assessment and weighing of the studies included in the meta-analyses to perform a sensitivity analysis.

\section{Certainty of evidence}

We will use Grading of Recommendations Assessment, Development, and Evaluations (GRADE) to assess the certainty of evidence. Using the GRADEpro tool we will construct a 'summary of findings' table for each outcome of the pooled studies. ${ }^{19}$

\section{Timelines for the review}

This protocol was written following the recommendations by the relevant PRISMA guidelines. ${ }^{20}$ We registered this study in the International Prospective Register of Systematic Reviews in October 2018. ${ }^{21}$ We planned to develop the search strategies, conduct preliminary searches, and pilot study selection processes between February and May 2019. We plan to finalise study selection, extract data from included studies, and conduct data analyses between June and December 2019; and prepare and submit the manuscript to a peer-reviewed journal by January 2020. 


\section{PATIENT AND PUBLIC INVOLVEMENT}

There was no patient or public involvement in the preparation of this protocol.

Contributors LM led the development of the protocol, wrote the first draft, coordinated and integrated comments from co-authors, approved the final version for publication, and is the guarantor of the manuscript. DEN and EP critically revised successive drafts of the manuscript, provided important intellectual input and approved the final version of the manuscript. CSW conceived the study, provided supervision and mentorship to LM, critically revised successive drafts of the manuscript, provided important intellectual input and approved the final version of the manuscript.

Funding This work is supported by the South African Medical Research Council (through salaries for LM, DEN, EP and CSW).

Competing interests None declared.

Patient consent for publication Not required.

Provenance and peer review Not commissioned; externally peer reviewed.

Open access This is an open access article distributed in accordance with the Creative Commons Attribution Non Commercial (CC BY-NC 4.0) license, which permits others to distribute, remix, adapt, build upon this work non-commercially, and license their derivative works on different terms, provided the original work is properly cited, appropriate credit is given, any changes made indicated, and the use is non-commercial. See: http://creativecommons.org/licenses/by-nc/4.0/.

\section{REFERENCES}

1. Holmes EC, Dudas G, Rambaut A, et al. The evolution of Ebola virus: Insights from the 2013-2016 epidemic. Nature 2016;538:193-200.

2. Zawilińska B, Kosz-Vnenchak M. General introduction into the Ebola virus biology and disease. Folia Med Cracov 2014;54:57-65.

3. Hoenen T, Groseth A, Feldmann H. Current ebola vaccines. Expert Opin Biol Ther 2012;12:859-72.

4. Bociaga-Jasik M, Piatek A, Garlicki A. Ebola virus disease pathogenesis, clinical presentation and management. Folia Med Cracov 2014;54:49-55.

5. Marcinkiewicz J, Bryniarski K, Nazimek K. Ebola haemorrhagic fever virus: pathogenesis, immune responses, potential prevention. Folia Med Cracov 2014;54:39-48.
6. Jadav SS, Kumar A, Ahsan MJ, et al. Ebola virus: current and future perspectives. Infect Disord Drug Targets 2015;15:20-31.

7. Lévy $\mathrm{Y}$, Lane $\mathrm{C}$, Piot $\mathrm{P}$, et al. Prevention of Ebola virus disease through vaccination: where we are in 2018. Lancet 2018;392:787-90.

8. Ebola vaccines - Background paper for SAGE deliberations. "Update with the development of Ebola vaccines and implications of emerging evidence to inform future policy recommendations". http:// www.who.int/immunization/sage/meetings/2018/october/2_Ebola_ SAGE20180ct_BgDoc_20180919.pdf?ua=1 [Accessed Dec 2018].

9. World Health Organisation. Ebola Virus "Ebola Viruses, E.V.D.W.H.O. 2018 http://www.who.int/biologicals/areas/vaccines/ebola_vaccines/ en/ [Accessed Aug 2018].

10. Marzi A, Feldmann H. Ebola virus vaccines: an overview of current approaches. Expert Rev Vaccines 2014;13:521-31.

11. Marzi A, Halfmann P, Hill-Batorski L, et al. Vaccines. An Ebola whole-virus vaccine is protective in nonhuman primates. Science 2015;348:aaa4919-442.

12. Stein RA. What is Ebola? Int J Clin Pract 2015;69:49-58.

13. Gross L, Lhomme E, Pasin C, et al. Ebola vaccine development: Systematic review of pre-clinical and clinical studies, and metaanalysis of determinants of antibody response variability after vaccination. Int $J$ Infect Dis 2018;74:83-96.

14. Kashangura R, Jullien S, Garner P, et al. MVA85A vaccine to enhance BCG for preventing tuberculosis. Cochrane Database Syst Rev 2019;4:CD012915.

15. Cochrane Effective Practice and Organisation of Care (EPOC). Suggested risk of bias criteria for EPOC reviews. EPOC Resources for review authors, 2017. 2017. http://epoc.cochrane.org/resources/ epoc-resources-review-authors

16. Sterne JA, Hernán MA, Reeves BC, et al. ROBINS-I: a tool for assessing risk of bias in non-randomised studies of interventions. BMJ 2016;355:i4919.

17. Review Manager (RevMan) [Computer program]. Version 5.3. Copenhagen: The Nordic Cochrane Centre, The Cochrane Collaboration, 2014.

18. Collaboration TC. In: Higgins JPT, Green S, eds. Cochrane handbook for systematic reviews of interventions version 5.1. 0, 2011.

19. GRADEpro, G. GRADEpro GDT: GRADEpro Guideline Development Tool [Software]. McMaster University, 2015 (developed by Evidence Prime, Inc. 2015. gradepro.org

20. Shamseer L, Moher D, Clarke M, et al. Preferred reporting items for systematic review and meta-analysis protocols (PRISMA-P) 2015: elaboration and explanation. BMJ 2015;349:g7647.

21. Mathebula L, Ndwandwe D, Pienaar E, et al. A systematic review of the effects of Ebola vaccines. PROSPERO 2018:CRD42018110505. http://www.crd.york.ac.uk/PROSPERO/display_record.php?ID= CRD42018110505 [Accessed 06 May 2019]. 